\title{
Time trends and disparities in anxiety among adolescents, 2012-2018
}

\author{
Katharine B. Parodi ${ }^{1} \cdot$ Melissa K. Holt ${ }^{1} \cdot$ Jennifer Greif Green ${ }^{1} \cdot$ Michelle V. Porche ${ }^{1} \cdot$ Brian Koenig $^{2} \cdot$ Ziming Xuan $^{3}$
}

Received: 1 October 2020 / Accepted: 2 June 2021 / Published online: 7 June 2021

(c) Springer-Verlag GmbH Germany, part of Springer Nature 2021

\begin{abstract}
Purpose Prior studies have been inconclusive in documenting whether the prevalence of adolescent anxiety is increasing, given sampling and measurement limitations. This study adds new information on recent time trends in anxiety prevalence, specifically investigating trends among previously unexamined sociodemographic subgroups.

Methods Weighted data of 37,360 youth respondents (51.1\% female, 71.8\% White, 91.3\% heterosexual, 99.2\% cisgender) from the 2012-2018 Dane County Youth Assessment, a county-wide survey administered to youth in participating school districts, were analyzed to estimate time trends in anxiety prevalence among the whole sample and by sociodemographic subgroups.

Results The prevalence of youth meeting anxiety-screening criteria increased from 34.1\% (95\% CI 33.4-34.9) in 2012 to $44 \%(95 \%$ CI 43.2-44.7) in 2018 (OR for trend $=1.07, P$ for trend $<0.001)$. The trend remained significant after adjusting for known confounds (AOR for trend $=1.07, P$ for trend $<0.001$ ). Anxiety increased significantly for several subgroups and widening disparities were documented among females relative to males $(P<0.001)$, and sexual minority youth relative to heterosexual youth $(P=0.003)$. In addition, Black youth did not increase at the same swift rate as White youth over the study period $(P<0.001)$.

Conclusion This study reports recent data on anxiety time trends and finds that among a geographically representative sample of adolescents, anxiety prevalence is rising. Findings provide new evidence documenting increased anxiety prevalence among sexual minority youth relative to their peers. Results highlight the need to bolster public health interventions focused on adolescent mental health, with tailored interventions for vulnerable groups.
\end{abstract}

Keywords Adolescence $\cdot$ Anxiety $\cdot$ Epidemiology $\cdot$ Health disparity $\cdot$ Time trend

\section{Introduction}

Anxiety is the most common mental health disorder among adolescents [1,2]. Nearly one-third experience a lifetime anxiety disorder, and $8.3 \%$ of youth meet criteria for severe anxiety-related impairment [2]. Left untreated, child and adolescent anxiety disorders often persist into adulthood and are associated with a range of adverse outcomes, including reduced educational attainment, adult psychopathology,

Melissa K. Holt

holtm@bu.edu

1 Department of Counseling Psychology and Applied Human Development, Boston University, Two Silber Way, Boston, MA 02215, USA

2 K12 Associates, Madison, WI, USA

3 Department of Community Health Sciences, Boston University, Boston, MA, USA substance use, premature mortality, lost productivity and increased healthcare expenditures [3-7]. Thus, enhanced understanding of trends and correlates of adolescent anxiety disorders is essential for prevention and intervention.

Recently, there has been considerable research discussion whether, and to what extent, mental health problems, including anxiety, might be increasing among youth $[8,9]$. Some evidence suggests that the prevalence of anxiety is rising among children, adolescents and young adults in the US [10-12], as well as in some European countries [13, 14]. For instance, one US nationally representative study found that from 2007 to 2011-2012, there was a 19\% increase for current anxiety among youth aged 6-17 years old [10]. Another study found evidence for cohort effects in reported symptoms of psychological distress, a related construct which includes anxiety and depressive symptoms, in the US and Canada [15]. Specifically, elevated scores of psychological distress were reported among the oldest (i.e., US individuals 
born 1912-1914) and youngest (i.e., Canadian adolescents and young adults born in the early- to mid-1990s) birth cohorts [15]. Similar findings emerged in a recent study of US adolescents and adults, with higher prevalence of psychological distress observed among the youngest generation [16].

However, several studies indicate that prevalence increases are limited to specific subpopulations [17, 18]. For example, a recent study of adolescent and young adult mental health in Great Britain revealed a small, but significant increase in psychological distress between 1991 and 2008, but only among females [18]. Similarly, a systematic review of twenty-first century time trends of youth mental health documented increases in internalizing symptoms among females in several studies, whereas findings for males were mixed [17].

The ability of researchers to answer basic surveillance questions about time trends is limited by inadequate data on anxiety trends. Studies described above use datasets that are many years old $[10,13,18]$, and rely on parental/caregiver report [10], which is known to be less effective than self-report in assessing anxiety among adolescents [19]. In addition, many studies have used measures of psychological distress which combine mood and anxiety symptoms, making it impossible to disaggregate time trends for anxiety [15-18]. As a result, further research is needed to determine whether anxiety has increased over the past decade and to inform prevention and intervention efforts.

In addition to determining whether the overall prevalence of anxiety is increasing, there are important reasons to identify whether time trends in anxiety differ for adolescent subpopulations. Disparities in anxiety prevalence are well established and indicate that females report higher anxiety prevalence than males $[1,2,20,21]$ and that anxiety is elevated among sexual and gender minority youth [22-24]. With respect to race and ethnicity, studies have been inconsistent in their findings regarding racial and ethnic differences in anxiety prevalence among youth [2, 25]. Importantly, few studies have examined anxiety time trends among subpopulations, with those that have examined differential time trends focusing primarily on age, sex, and/or racial and ethnic differences [11-13].

The present study contributes to the emerging body of literature investigating whether anxiety is increasing among adolescents, and for whom, and addresses some of the limitations of previous studies using an empirically validated self-report anxiety measure in a large-scale, geographically representative, and sociodemographically diverse sample of US adolescents. Specifically, this study uses repeated crosssectional data from youth surveys to examine: (1) trends in the prevalence of anxiety among adolescents, generally, and (2) trends in the prevalence of anxiety among adolescent sociodemographic subgroups. We examine subgroups for which time trends have been examined in prior research (sex and race/ethnicity), but also provide new information on whether anxiety is increasing among sexual minority youth and transgender youth. While prior studies have found heightened anxiety prevalence among sexual minority and transgender youth as compared to their peers at one time point [22-24], these studies have not examined whether time trends in anxiety are equivalent for sexual and gender minority youth. Specifically, it is unclear whether recent increased support for sexual and gender minority youth by society and key pieces of legislation might be associated with a reduced increase in anxiety prevalence, as compared to their peers. Increased understanding of recent time trends, and specifically trends for vulnerable groups, is essential for informing public health efforts and specifying points for prevention and intervention.

\section{Methods}

\section{Study population}

The Dane County Youth Assessment (DCYA) is a cross-sectional, web-based survey administered every 3 years to middle and high school students in Wisconsin's Dane County [26]. The county is the second most populous in this Midwestern state (population estimate of 542,364; 50.4\% female; 84\% White, 5.3\% Black or African American, 0.4\% American Indian and Alaska Native, 5.9\% Asian, $0.8 \%$ another race, $3.5 \%$ two or more races; in terms of ethnicity, $6.4 \%$ identified as Hispanic or Latino [27]). The county encompasses a geographically diverse region of urban, suburban and rural school districts. Traditional high school enrollment in the US is for students in grades 9 through 12, and the age correspondence for these grades is generally between 14 and 18 years. The high school student enrollment in school districts ranged from approximately 22,000 to 24,000 students across the 2011-2012 and 2017-2018 academic years [28]. All school districts located in Dane County were invited to participate across survey years. An additional school district, partially in the county, was included beginning with the 2015 survey year. Over the study period considered, only one public high school in 2012 and two public high schools in 2015 chose not to participate. The current study represents an analysis of the 2012, 2015 and 2018 high school data. At each survey administration, all school districts used a passive parental consent form (i.e., parents/caregivers sent back forms only if they wanted to opt their student out of survey participation), and youth provided assent. Response rates were high across survey years ( $>90 \%$ for most school districts in 2012 and 2015; > 85\% in 2018). All students were surveyed in the majority of schools; however, in large urban schools, approximately $50 \%$ of students were sampled 
by design in each survey year. Surveys included questions about sociodemographic characteristics, health behaviors, and perceptions of family, school, and community issues. A measure of anxiety was introduced in the 2012 survey administration. In 2012, 14,158 students in 22 high schools completed the survey. In 2015, 13,905 students in 22 high schools and 1 juvenile detention facility completed the survey. In 2018, 16,288 students in 24 high schools participated. Given high participation rates across survey years, the percentage of participating schools in the county, and the complex survey design used in the large urban schools, survey results are considered representative of the population. The Institutional Review Board at Boston University declared this study exempt human subjects research.

\section{Participants}

Students reported their grade in school (9th-12th), sex (male/female), race/ethnicity (White, Black, Hispanic or Latino, Hmong, Asian, Native, Middle Eastern, Multiracial), sexual orientation (heterosexual/gay or lesbian/ bisexual/questioning), and gender identity [transgender/not transgender (researcher-classified as cisgender)] as detailed in Table 1. Given some sociodemographic items (e.g., race/ ethnicity) included slightly different response options across survey years, analyses were restricted to common response options in the dataset. In the overall sample, the weighted proportions by sociodemographic characteristics were $51.1 \%$ female, $71.8 \%$ White, $91.3 \%$ heterosexual, and $99.2 \%$ cisgender, and the mean age was $15.9(S D=1.2)$.

\section{Measures}

\section{Anxiety}

The study used a modified version of the Generalized Anxiety Disorder-2 (GAD-2) to assess adolescent anxiety [29]. The GAD-2 assesses anxiety symptoms in the past 2 weeks with items rated on a 4-point Likert-type scale. It is considered a brief, valid and reliable screening measure [29-31], and has specifically shown good internal consistency reliability in adolescent samples [32]. In the DCYA, the GAD-2
Table 1 Sociodemographic characteristics of Dane County Youth Assessment respondents

\begin{tabular}{|c|c|c|c|c|c|c|}
\hline & \multicolumn{2}{|l|}{2012} & \multicolumn{2}{|l|}{2015} & \multicolumn{2}{|l|}{2018} \\
\hline & $n$ & $\%$ & $n$ & $\%$ & $n$ & $\%$ \\
\hline \multicolumn{7}{|l|}{ Grade } \\
\hline 9th & 3165 & 26.2 & 3507 & 29.4 & 3791 & 28.6 \\
\hline 10th & 3077 & 25.6 & 3192 & 27.8 & 3593 & 27.1 \\
\hline 11 th & 2944 & 25.8 & 2918 & 23.6 & 3375 & 24.8 \\
\hline 12 th & 2625 & 22.4 & 2460 & 19.2 & 2713 & 19.5 \\
\hline \multicolumn{7}{|l|}{ Sex } \\
\hline Male & 5766 & 48.8 & 5994 & 49.0 & 6582 & 49.2 \\
\hline Female & 6045 & 51.2 & 6083 & 51.0 & 6890 & 50.8 \\
\hline \multicolumn{7}{|l|}{ Race/ethnicity } \\
\hline White & 8997 & 74.6 & 9520 & 71.0 & 10,002 & 68.9 \\
\hline Black & 631 & 6.2 & 550 & 8.1 & 593 & 7.5 \\
\hline Hispanic or Latino & 619 & 6.0 & 620 & 8.3 & 964 & 10.1 \\
\hline Hmong & 194 & 1.7 & 139 & 1.8 & 194 & 1.6 \\
\hline Asian & 331 & 2.9 & 385 & 3.5 & 561 & 3.9 \\
\hline Native & 69 & 0.6 & 69 & 0.5 & 87 & 0.5 \\
\hline Middle Eastern & 69 & 0.6 & 50 & 0.5 & 52 & 0.4 \\
\hline Multi-racial & 901 & 7.4 & 744 & 6.3 & 1019 & 7.1 \\
\hline \multicolumn{7}{|l|}{ Sexual orientation } \\
\hline Heterosexual & 11,111 & 93.6 & 11,155 & 91.5 & 12,047 & 89.1 \\
\hline Gay or lesbian & 118 & 1.1 & 173 & 1.5 & 233 & 1.8 \\
\hline Bisexual & 364 & 3.4 & 495 & 4.7 & 831 & 6.4 \\
\hline Questioning & 218 & 1.9 & 254 & 2.3 & 361 & 2.7 \\
\hline \multicolumn{7}{|l|}{ Gender identity } \\
\hline Cisgender & 11,675 & 98.8 & 11,986 & 99.3 & 13,381 & 99.3 \\
\hline Transgender & 136 & 1.2 & 91 & 0.7 & 91 & 0.7 \\
\hline
\end{tabular}

Unweighted number of respondents and weighted percentage of respondents are reported Because of rounding, percentages may not total 100 
was modified to ask about past-month symptoms. Specifically, students were asked: in the past month, how often have you been bothered by the following problems? (1) Feeling nervous, anxious, or on edge, and (2) Not being able to stop or control worrying. The response options for the DCYA were: $1=$ Always, $2=$ Often, $3=$ A little, $4=$ Not at all. Responses were re-coded from 4, 3, 2, 1 into 0, 1, 2, 3 for each item to correspond with the GAD-2 scoring. The two items were summed to match the $0-6$ subscale range of the GAD-2 measure, with higher scores indicating more probable anxiety. Using the optimal cut-point of $\geq 3$ on the GAD-2 among general samples of the population [29-31], a binary indicator variable was created to reflect anxious/ not anxious.

\section{Depression}

One item assessed past-year depression symptomology in the DCYA, with students asked: during the past 12 months, did you ever feel so sad or hopeless almost every day for at least 2 weeks in a row that you stopped doing some usual activities? Response options for this item were $1=$ Yes, $2=N o$. Response options for this variable were re-coded to $1=$ Not depressed, $2=$ Depressed in the data frame, and this variable was treated as a covariate in adjusted regression analyses due to well-established comorbidity between anxiety and depression in the literature [33, 34].

\section{Analytic strategy}

All analyses were conducted in R version 3.6.0 [35] and followed a similar analytic strategy to a depression time trends study by Weinberger et al. [36]. Open-source code for the present study is available at https://github.com/katharinep arodi/dcya-anxiety-sppe. To assess anxiety trends, an index measure of year was created to represent the survey year, ranging from 1 for 2012 to 7 for 2018. Consistent with prior DCYA research [37, 38], a screener using extreme height and weight responses and multiple low-frequency items was implemented to identify and exclude 314 cases of potentially mischievous respondents, and the final analytic sample represents exclusion of these cases. Analyses used the DCYA sampling weights to ensure population-based representativeness; sampling weights account for the racial/ethnic composition of participant schools. Trends analyses employed multiple year weight adjustment depending on the number of times a school participated in the survey. In the analytic sample, $10.2 \%$ of respondents were missing responses on any study variable. The outcome variable had $4.9 \%$ missingness, sociodemographic variables ranged from 0.8 to $2.5 \%$ missingness, and the depression variable had $5.8 \%$ missingness. Subsequently, a complete-case analysis was conducted on an analytic sample of 37,360 respondents.
The prevalence and associated $95 \%$ confidence intervals of all youth respondents meeting GAD-2 screening criteria were calculated for each survey year in the merged dataset. Data were stratified by grade, sex, race/ethnicity, sexual orientation, and gender identity, with the prevalence and 95\% confidence intervals calculated for each subgroup for each survey year. To test significance of the time trend among the aggregate sample, a logistic regression model predicting anxiety, with survey year as continuous predictor, was estimated in an unadjusted model. An adjusted model was fit by adding grade, sex, race/ethnicity, sexual orientation, gender identity, and depression variables simultaneously into the model. For subgroup analyses, unadjusted and adjusted stratified logistic regression models predicting anxiety, with survey year as continuous predictor, were estimated to test time trends. Logistic regression models predicting anxiety tested the interaction of each sociodemographic variable and survey year in fully adjusted models to examine if trends varied within subgroups. In all models, we used a sandwich estimator approach of generalized linear models to obtain robust standard error estimates to account for the clustering of students within schools.

\section{Results}

\section{Prevalence and trends in anxiety}

Estimated prevalence results by survey year varied considerably among the sample and by sociodemographic characteristics (Table 2). Analyses revealed that $34.1 \%$ [confidence interval (CI) 33.4-34.9] of all adolescent respondents met screening criteria for anxiety in 2012. Higher prevalence estimates were documented in 2015 and 2018, with $37.7 \%$ (CI 36.9-38.4) and 44\% (CI 43.2-44.7) of youth meeting screening criteria for anxiety, respectively (Fig. 1a). Both unadjusted and adjusted logistic regression models indicated that past-month anxiety increased significantly between 2012 and 2018.

\section{Prevalence and trends in anxiety by sociodemographic characteristics}

\section{Grade}

Anxiety increased among students in each of grades 9 through 12 over the survey period (Fig. 1b). Increases were significant in both unadjusted and adjusted models (Table 2). The interaction between survey year and grade revealed no significant difference in the rate of increase among students in grades 10, 11 and 12 compared to grade 9 (Table 3 ). 
Table 2 Estimated prevalence and trends of past-month anxiety in the sample and stratified by sociodemographic characteristics

\begin{tabular}{|c|c|c|c|c|c|c|c|}
\hline & \multicolumn{3}{|c|}{ Prevalence estimates $[95 \% \mathrm{CI}]$} & \multicolumn{4}{|c|}{ Test of temporal trend } \\
\hline & 2012 & 2015 & 2018 & OR $[95 \% \mathrm{CI}]^{\mathrm{a}}$ & $p$ value & $\operatorname{AOR}[95 \% \mathrm{CI}]^{\mathrm{b}}$ & $p$ value \\
\hline All participants & $34.1[33.4,34.9]$ & $37.7[36.9,38.4]$ & $44.0[43.2,44.7]$ & $1.07[1.06,1.09]$ & $<0.001$ & $1.07[1.05,1.09]$ & $<0.001$ \\
\hline \multicolumn{8}{|l|}{ Grade } \\
\hline 9 th & $30.4[29.0,31.8]$ & $34.0[32.7,35.4]$ & $38.7[37.3,40.1]$ & $1.06[1.04,1.09]$ & $<0.001$ & $1.07[1.03,1.10]$ & $<0.001$ \\
\hline 10th & $34.8[33.3,36.3]$ & $40.1[38.7,41.6]$ & $43.6[42.2,45.0]$ & $1.06[1.04,1.08]$ & $<0.001$ & $1.06[1.04,1.08]$ & $<0.001$ \\
\hline 11 th & $35.5[34.0,37.0]$ & $37.5[36.0,39.0]$ & $47.6[46.1,49.1]$ & $1.09[1.06,1.12]$ & $<0.001$ & $1.07[1.04,1.10]$ & $<0.001$ \\
\hline 12 th & $36.3[34.7,37.9]$ & $39.8[38.1,41.5]$ & $47.7[46.0,49.4]$ & $1.08[1.06,1.11]$ & $<0.001$ & $1.09[1.05,1.12]$ & $<0.001$ \\
\hline \multicolumn{8}{|l|}{ Sex } \\
\hline Male & $26.0[25.0,27.0]$ & $24.1[23.1,25.0]$ & $31.3[30.3,32.3]$ & $1.05[1.02,1.07]$ & $<0.001$ & $1.04[1.02,1.06]$ & $<0.001$ \\
\hline Female & $41.9[40.8,43.0]$ & $50.7[49.6,51.8]$ & $56.2[55.2,57.3]$ & $1.10[1.08,1.12]$ & $<0.001$ & $1.09[1.07,1.11]$ & $<0.001$ \\
\hline \multicolumn{8}{|l|}{ Race/ethnicity } \\
\hline White & $34.0[33.2,34.9]$ & $37.2[36.3,38.1]$ & $43.7[42.8,44.6]$ & $1.07[1.05,1.09]$ & $<0.001$ & $1.08[1.06,1.09]$ & $<0.001$ \\
\hline Black & $33.7[30.8,36.7]$ & $38.0[35.4,40.7]$ & $36.2[33.7,38.9]$ & $1.02[0.98,1.06]$ & 0.372 & $0.99[0.95,1.03]$ & 0.670 \\
\hline Hispanic or Latino & $32.0[29.1,35.1]$ & $36.2[33.6,38.8]$ & $46.0[43.6,48.4]$ & $1.11[1.07,1.15]$ & $<0.001$ & $1.09[1.07,1.12]$ & $<0.001$ \\
\hline Hmong & $36.6[30.9,42.7]$ & $44.6[38.9,50.5]$ & $43.2[37.5,49.1]$ & $1.04[0.99,1.10]$ & 0.136 & $1.02[0.92,1.11]$ & 0.743 \\
\hline Asian & $37.1[32.7,41.7]$ & $35.4[31.5,39.4]$ & $46.2[42.5,50.0]$ & $1.08[1.04,1.11]$ & $<0.001$ & $1.09[1.04,1.15]$ & 0.001 \\
\hline Native & $32.3[23.5,42.7]$ & $34.8[25.2,45.8]$ & $51.8[41.0,62.5]$ & $1.13[1.01,1.24]$ & 0.041 & $1.11[0.96,1.26]$ & 0.178 \\
\hline Middle Eastern & $33.4[24.7,43.3]$ & $52.4[41.9,62.7]$ & $53.4[41.3,65.1]$ & $1.15[1.03,1.27]$ & 0.018 & $1.27[1.12,1.43]$ & 0.002 \\
\hline Multi-racial & $35.6[32.9,38.4]$ & $42.5[39.4,45.5]$ & $50.0[47.2,52.9]$ & $1.10[1.06,1.15]$ & $<0.001$ & $1.07[1.02,1.11]$ & 0.011 \\
\hline \multicolumn{8}{|l|}{ Sexual orientation } \\
\hline Heterosexual & $32.2[31.5,33.0]$ & $34.8[34.1,35.6]$ & $40.5[39.7,41.2]$ & $1.06[1.05,1.08]$ & $<0.001$ & $1.07[1.05,1.08]$ & $<0.001$ \\
\hline Gay or lesbian & $52.2[44.6,59.6]$ & $63.3[57.2,69.1]$ & $72.0[66.8,76.7]$ & $1.15[1.08,1.22]$ & $<0.001$ & $1.20[1.12,1.27]$ & $<0.001$ \\
\hline Bisexual & $63.7[59.5,67.7]$ & $68.8[65.3,72.0]$ & $73.1[70.4,75.7]$ & $1.08[1.02,1.13]$ & 0.005 & $1.08[1.03,1.13]$ & 0.002 \\
\hline Questioning & $64.4[58.7,69.6]$ & $69.8[64.9,74.3]$ & $72.1[67.8,76.0]$ & $1.06[1.00,1.12]$ & 0.045 & $1.04[0.98,1.10]$ & 0.231 \\
\hline \multicolumn{8}{|l|}{ Gender identity } \\
\hline Cisgender & $34.0[33.2,34.7]$ & $37.5[36.7,38.2]$ & $43.8[43.1,44.6]$ & $1.07[1.06,1.09]$ & $<0.001$ & $1.07[1.05,1.09]$ & $<0.001$ \\
\hline Transgender & $48.9[41.8,56.1]$ & $66.2[56.9,74.3]$ & $66.0[56.8,74.2]$ & $1.14[1.07,1.21]$ & $<0.001$ & $1.07[0.95,1.19]$ & 0.299 \\
\hline
\end{tabular}

Data Source: The Dane County Youth Assessment

CI Confidence Interval, OR Odds Ratio, AOR Adjusted Odds Ratio

a The odds of meeting screening criteria for past-month anxiety versus not meeting screening criteria

${ }^{b}$ The odds of meeting screening criteria for past-month anxiety versus not meeting screening criteria, controlling for all other covariates

\section{Sex}

The estimated prevalence and temporal changes in youth meeting screening criteria for anxiety varied by sex (Fig. 1c). The percentage of females meeting GAD-2 screening criteria significantly increased from $41.9 \%$ (CI $40.8-43.0$ ) in 2012 to $50.7 \%$ (CI 49.6-51.8) in 2015 to $56.2 \%$ (CI 55.2-57.3) in 2018. Prevalence for males fluctuated across survey years. In 2012, $26 \%$ (CI 25.0-27.0) of males met anxiety-screening criteria, with prevalence decreasing to $24.1 \%$ (CI 23.1-25.0) in 2015, and increasing to $31.3 \%$ (CI 30.3-32.3) in 2018, a significant temporal change (Table 2). The interaction between survey year and sex was significant and revealed females had a steeper rate of increase than males (Table 3).

\section{Race/ethnicity}

Significant upward trends in anxiety were observed among Hispanic/Latino, Middle Eastern, Multi-racial, and White youth in both unadjusted and adjusted models (Fig. 1d). Hispanic/Latino youth increased from $32 \%$ (CI 29.1-35.1) in 2012 to $36.2 \%$ (CI 33.6-38.8) in 2015, before rising sharply to $46 \%$ (CI 43.6-48.4) in 2018. A steep increase was observed among Middle Eastern youth, who increased from $33.4 \%$ (CI 24.7-43.3) in 2012 to $52.4 \%$ (CI 41.9-62.7) in 2015 to $53.4 \%$ (CI 41.3-65.1) in 2018. Multi-racial youth increased from $35.6 \%$ (CI $32.9-38.4$ ) in 2012 to $42.5 \%$ (CI 39.4-45.5) in 2015 to $50 \%$ (CI 47.2-52.9) in 2018. White youth evidenced a large increase in youth meeting anxiety-screening criteria 
(a)

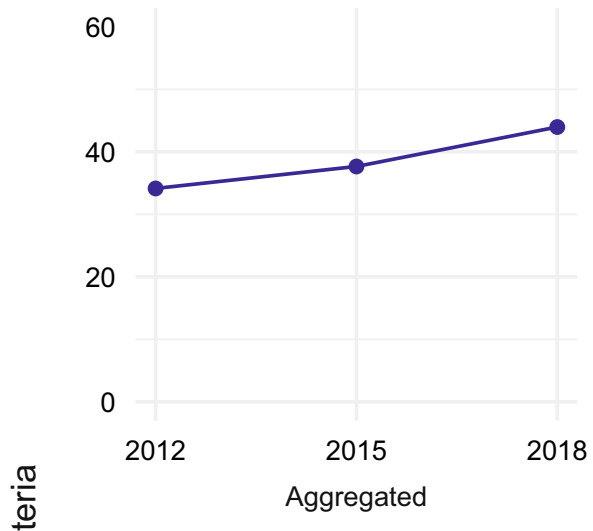

(c)

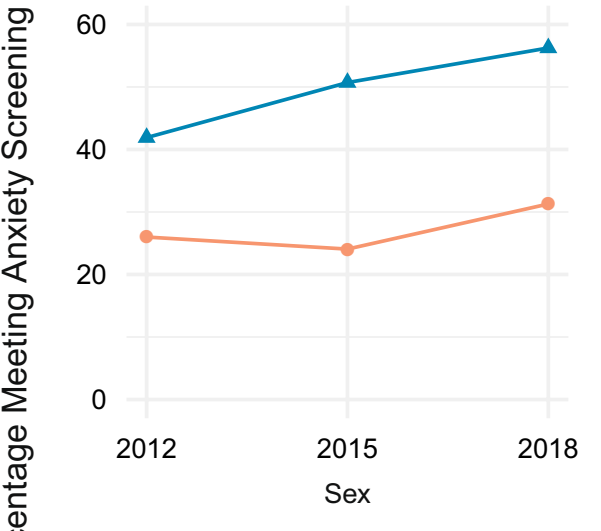

(e)

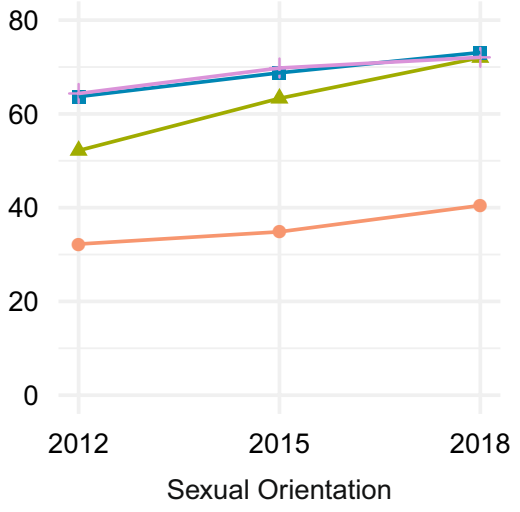

(b)

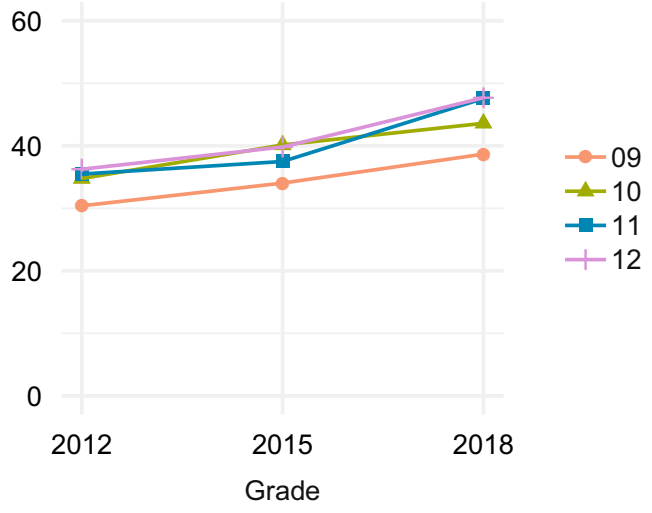

(d)

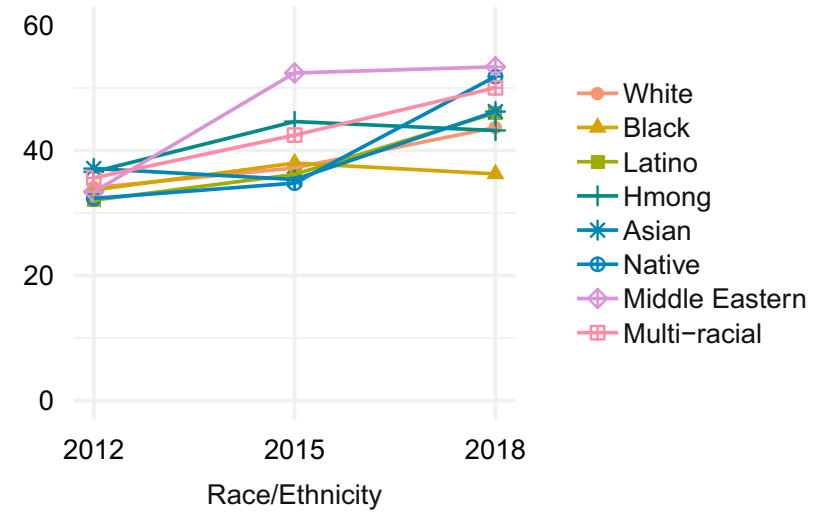

(f)

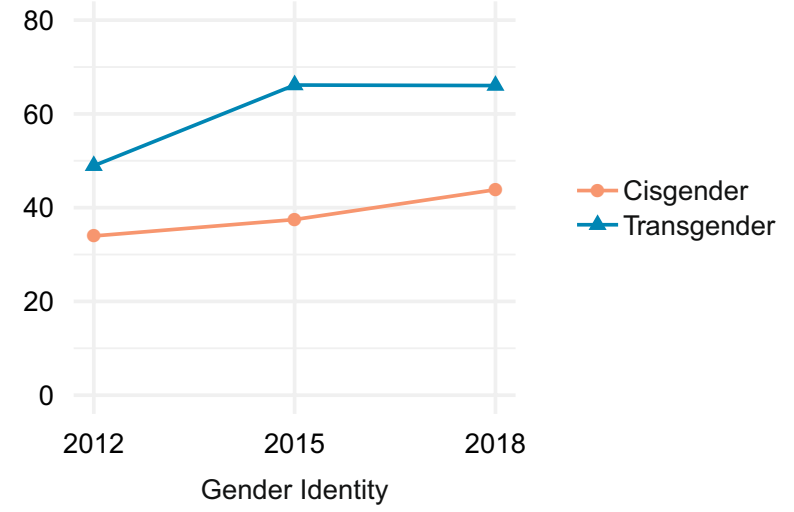

Fig. 1 Time trend of past-month anxiety symptoms for $\mathbf{a}$ all adolescent participants, $\mathbf{b}$ by grade level, $\mathbf{c}$ by sex, $\mathbf{d}$ by race/ethnicity, $\mathbf{e}$ by sexual orientation, $\mathbf{f}$ by gender identity. DCYA Dane County Youth Assessment

across the survey period, rising from $34 \%$ (CI 33.2-34.9) in 2012 to $43.7 \%$ (CI 42.8-44.6) in 2018.

Prevalence estimates of anxiety for Native youth significantly increased from $32.3 \%$ (CI 23.5-42.7) to $34.8 \%$ (CI $25.2-45.8$ ) to $51.8 \%$ (CI 41.0-62.5) across survey years in the unadjusted model. Anxiety prevalence among Asian youth decreased from $37.1 \%$ (CI 32.7-41.7) in 2012 to $35.4 \%$ (CI 31.5-39.4) in 2015 before rising to $46.2 \%$ (CI 42.5-50.0) in 2018, a significant trend in both unadjusted and adjusted models. The interaction between survey year and race/ethnicity was significant for Black compared to White youth, whereby Black youth did not increase at the same steep rate as White youth over survey years (Table 3).

\section{Sexual orientation}

The percentage of heterosexual youth meeting GAD-2 screening criteria exhibited a significant upward trend, 
Table 3 Logistic regression results of interaction models of sociodemographic factors and trend

\begin{tabular}{|c|c|c|}
\hline & Interacti & h trend \\
\hline & $z$ value & $p$ value \\
\hline Grade & & \\
\hline 9th (Reference) & & \\
\hline 10 th & -0.20 & 0.842 \\
\hline 11 th & 0.57 & 0.571 \\
\hline 12 th & 0.97 & 0.334 \\
\hline Sex & & \\
\hline Male (Reference) & & \\
\hline Female & 3.44 & $<.001$ \\
\hline Race/ethnicity & & \\
\hline White (Reference) & & \\
\hline Black & -4.94 & $<.001$ \\
\hline Hispanic or Latino & 1.07 & 0.286 \\
\hline Hmong & -1.19 & 0.235 \\
\hline Asian & 0.46 & 0.647 \\
\hline Native & 0.19 & 0.846 \\
\hline Middle Eastern & 1.37 & 0.170 \\
\hline Multi-racial & -0.22 & 0.823 \\
\hline Sexual orientation & & \\
\hline Heterosexual (Refe & & \\
\hline Gay or lesbian & 3.02 & 0.003 \\
\hline Bisexual & 0.43 & 0.664 \\
\hline Questioning & -1.15 & 0.252 \\
\hline Gender identity & & \\
\hline Cisgender (Referen & & \\
\hline Transgender & -0.55 & 0.584 \\
\hline
\end{tabular}

Data Source: The Dane County Youth Assessment

increasing from $32.2 \%$ (CI $31.5-33.0$ ) in 2012 to $34.8 \%$ (CI 34.1-35.6) in 2015 to 40.5\% (CI 39.7-41.2) in 2018 (Fig. 1e). Unadjusted and adjusted analyses revealed a significant increase in anxiety among gay or lesbian and bisexual youth. In 2012, 52.2\% (CI 44.6-59.6) of gay or lesbian youth and $63.7 \%$ (CI 59.5-67.7) of bisexual youth met anxiety-screening criteria. By 2018, prevalence estimates increased to $72 \%$ (CI 66.8-76.7) for gay or lesbian youth and $73.1 \%$ (CI 70.4-75.7) for bisexual youth, respectively. In the unadjusted model, a significant increase among youth questioning their sexual orientation was observed; however, this upward trend no longer remained significant in the adjusted model (Table 2). The interaction between survey year and sexual orientation revealed a more rapid increase in anxiety among gay or lesbian youth compared to heterosexual youth (Table 3 ).

\section{Gender identity}

The estimated prevalence of anxiety among cisgender youth significantly increased from 34\% (CI 33.2-34.7) in 2012 to $37.5 \%$ (CI 36.7-38.2) in 2015 and to $43.8 \%$ (CI 43.1-44.6) in 2018 (Fig. 1f). The estimated prevalence of anxiety among transgender youth increased from $48.9 \%$ (41.8-56.1) in 2012 to $66.2 \%$ (CI 56.9-74.3) in 2015, and remained at $66 \%$ (CI 56.8-74.2) in 2018. While this trend was significant in the unadjusted model, it became non-significant after controlling for covariates (Table 2). The interaction between survey year and gender identity was not significant (Table 3 ).

\section{Discussion}

This study documents an increase in anxiety among high school students in several large, diverse school districts in a Midwestern US county from 2012 to 2018. Overall, analyses revealed a $29 \%$ increase in youth meeting screening criteria for past-month anxiety over the study period. While few studies of recent time trends in youth anxiety exist, our findings are consistent with a small set of studies documenting that anxiety is increasing among youth and emerging adults in the United States [10-12] and also with evidence of the increasing prevalence of related mental health conditions and symptoms (e.g., depressive symptoms, suicidal thoughts and behaviors) and treatment use among youth and young adults nationally [36, 39-42]. In regards to the latter, our findings align with recent evidence for increased service utilization provided by office-based physicians for youth experiencing anxiety disorders [42].

Examining anxiety trends by sociodemographic characteristics revealed increases in anxiety for particular adolescent subgroups, as well as increasing disparities among a smaller set of subgroups. Notably, females had rising rates of anxiety over survey years compared to males, a finding consistent with recent research documenting increases in psychological distress among a representative sample of adolescents and young adults ages 16-24 in Great Britain [18]. In addition, our finding aligns with evidence for sex differences in the increasing prevalence of depression-a highly comorbid disorder-among adolescent females compared to males [40]. In particular, Keyes et al. recently documented that depressive symptoms rose significantly over the same time period (2012-2018) in a nationally representative sample of US school-attending youth, with marked increases among females [39]. Grade level also emerged as an important epidemiological factor, with anxiety increasing for students in each grade level between 2012 and 2018 in stratified analyses.

Results documented considerable variation in the estimated prevalence of anxiety across racial and ethnic groups. 
Prior studies found that Black youth report higher prevalence of anxiety compared to their racial and ethnic counterparts [2], and Black males, in particular, report elevated physiologic anxiety [25]. In contrast, we found that Black youth reported lower prevalence of anxiety in 2012 and 2018 compared to White youth, and that Black youth did not increase at the swift rate documented among White youth in the sample. Given differences in the measures employed to assess anxious symptomology across comparable studies (e.g., McLaughlin and colleagues used the Multidimensional Anxiety Scale for Children which includes a physical symptoms subscale), including items which measure physiological symptoms of anxiety among youth is warranted for future studies. In addition, increases in anxiety were evident among Multi-racial, Middle Eastern and Hispanic/Latino youth, pointing to the need for further research on individual and contextual factors identified by adults and youth of color in the United States as contributing to elevated stress [43] and potentially to prevalence changes in anxiety symptoms. In particular, more research into adolescent subpopulations impacted by racial and ethnic discrimination is needed given the link between this stressor and internalizing symptomsincluding anxiety—among adolescents [44].

Additional subgroup differences revealed two subgroups with particular anxiety risk: lesbian, gay, bisexual or questioning (LGBQ) and transgender youth. Notably, LGBQ youth had high prevalence $(>50 \%)$ of anxiety across all time points. Youth who identified as gay or lesbian also reported more rapidly increasing anxiety than their heterosexual peers. While the trend analysis for youth identifying as transgender was not significant in the adjusted analysis, high prevalence estimates of anxiety were observed at each time point (e.g., 68.4\% in 2015). These results align with findings that sexual and gender minority youth experience high prevalence of psychological distress, including mood and anxiety disorders, as well as disparities in their mental health compared to their heterosexual and cisgender counterparts, respectively [22, 45-47]. Results are also consistent with a finding that $21 \%$ of Generation $\mathrm{Z}$ youth (those born between 1997 to present) report issues related to their sexual orientation or gender identity as a source of stress [43]. Further research examining proximal and distal contributors to these differences is warranted.

\section{Limitations}

While this study advances the literature on recent trends and sociodemographic differences in anxiety among youth, limitations exist. First, the sample is from one county, which limits the generalizability of the findings. Second, while the administration of the survey over regular intervals permits trends analyses, the study may be impacted by the specific period of data collection. In addition, although analyses were restricted to the high school data, some students may have completed the survey in multiple years and no identifier was available to remove students completing multiple surveys. To address this limitation, post hoc analyses were conducted in which grade 9 was removed across survey years, as the 3-year gap in survey administration makes it likely that 9 th graders completed the survey again as 12 th graders. Removing grade 9, the time trend among the entire sample remained significant across models (Supplementary Table 1). In stratified models, the significance of all subgroup temporal trends remained the same, except for differences in the unadjusted and adjusted analyses among Hmong and Multi-racial youth, respectively, and for the unadjusted models for transgender and questioning youth becoming non-significant when grade 9 was removed (Supplementary Table 1). The interaction between survey year and sexual orientation also indicated that questioning youth were increasing in anxiety at a swifter rate than heterosexual youth with grade nine removed (Supplementary Table 2).

Another limitation is related to the outcome measure. Although the GAD-2 is an empirically validated measure, it requires more psychometric research in adolescent samples and is a brief screening tool that relies on self-report of past anxiety symptoms versus a measure such as the Pediatric Anxiety Rating Scale (PARS) which is administered by a clinician. Our analyses used a slightly modified version of the GAD-2 which assessed past-month (and not past 2 weeks) symptoms of anxiety, as well as slight variation in response scale wording. Importantly, high anxiety prevalence estimates were documented across the study period, and it is critical to note that use of the GAD-2 screening instrument only reflects trends in those meeting the clinical threshold in self-reported anxiety symptoms and does not represent those meeting diagnostic criteria.

There is limited information on the validity of selfreport assessments of psychological symptoms for several subgroups included in this study. No measure of socioeconomic status was included in the present study, though it has known interactions with race/ethnicity in its association with mental health [48, 49]. In addition, while a strength of the study is the large sample size, low power to detect effects in the transgender subsample is a limitation. In the future, additional time points from the DCYA data could be incorporated to assess longer term trends. Longitudinal research would allow for consideration of not only time trends in anxiety, but also associated outcomes. Lastly, it is unclear if rising anxiety prevalence is due to greater incidences of anxiety among adolescents or increased disclosure of symptoms among younger cohorts of youth.

Further research could build upon this study using national datasets to explore whether findings are replicated in a national sample of US adolescents, or if results are specific to the geographic region sampled. Future work should 
examine other anxiety symptoms common in youth (e.g., symptoms of social anxiety disorder) using more comprehensive measures, as well as clarify contributions of other comorbid conditions (e.g., substance use) in addition to the depression item included in the present analyses. Future studies should elucidate modifiable factors at the individualand contextual-level of analysis contributing to increases in anxiety. Relatedly, further investigation into factors driving differential trends among specific subgroups is warranted to improve youth mental health within the subgroup populations.

\section{Conclusion}

Anxiety is a prevalent mental health condition among adolescents, which often goes undiagnosed and untreated. Results from this study documented increases in youth meeting screening criteria for anxiety in several large, diverse school districts between 2012 and 2018. In addition, increasing disparities in anxiety prevalence were noted among specific subgroups (i.e., females, gay or lesbian youth). Results point to a need for further research and policy efforts to address the rising prevalence of anxiety and widening mental health disparities observed. Such research is particularly needed in light of shifting patterns in youth and young adult mental health due to the global COVID-19 pandemic $[50,51]$. Studies addressing the concurrent patterning of documented social determinants of mental health (e.g., discrimination, violence victimization, financial stressors) and their potential link to recent changes in anxiety prevalence are needed to identify key factors driving these patterns. Increased knowledge of such factors may provide important opportunities to directly address root causes of anxiety uptrend through multilevel interventions [52].

In addition, effective health care and educational policies are needed to improve detection and access to care for youth in diverse settings (e.g., schools, clinics), especially among groups with known barriers to care. Universal school-based mental health screening provides an important approach for identifying youth at risk for anxiety disorders and connecting them with school-based supports and mental health services. Relatedly, given the critical role psychiatrists, as well as primary care providers serve in identifying and treating youth with anxiety disorders [42], policy initiatives can promote collaborative efforts among these medical specialties. Lastly, given that Hispanics/Latinos are the largest US ethnic minority [53], future studies should attend to the significant upward trends observed among specific populations in the US, as well as the critical questions about mental health patterns and health equity posed by these sociodemographic increases.
Supplementary Information The online version contains supplementary material available at https://doi.org/10.1007/s00127-021-02122-9.

Funding Funding for this project was supported by the Dane County Youth Commission, the Dane County Department of Human Services, the United Way of Dane County, the Public Health Department of Madison and Dane County, and the participating Dane County School Districts.

Availability of data and material Additional information on the data source, the Dane County Youth Assessment, is available at: https:// www.danecountyhumanservices.org/yth/default.aspx

Code availability Open-source code for this study is available at https:// github.com/katharineparodi/dcya-anxiety-sppe.

\section{Declarations}

Conflict of interest On behalf of all the authors, the corresponding author states that there is no conflict of interest.

Ethical approval The study has been approved by the appropriate ethics committee and has, therefore, been performed in accordance with the ethical standards laid down in the 1964 Declaration of Helsinki and its later amendments.

\section{References}

1. Kessler RC, Avenevoli S, Costello EJ et al (2012) Prevalence, persistence, and sociodemographic correlates of DSM-IV disorders in the National comorbidity survey replication adolescent supplement. Arch Gen Psychiatry 69(4):372-379. https://doi.org/ 10.1001/archgenpsychiatry.2011.160

2. Merikangas KR, He J, Burstein M et al (2010) Lifetime prevalence of mental disorders in U.S. adolescents: results from the National Comorbidity Survey Replication-Adolescent Supplement (NCSA). J Am Acad Child Adolesc Psychiatry 49(10):980-989. https:// doi.org/10.1016/j.jaac.2010.05.017

3. Mojtabai R, Stuart EA, Hwang I, Eaton WW, Sampson N, Kessler RC (2015) Long-term effects of mental disorders on educational attainment in the National Comorbidity Survey ten-year followup. Soc Psychiatry Psychiatr Epidemiol 50(10):1577-1591. https://doi.org/10.1007/s00127-015-1083-5

4. Essau CA, Lewinsohn PM, Olaya B, Seeley JR (2014) Anxiety disorders in adolescents and psychosocial outcomes at age 30 . $\mathrm{J}$ Affect Disord 163(C):125-132. https://doi.org/10.1016/j.jad.2013. 12.033

5. Lopez B, Turner RJ, Saavedra LM (2005) Anxiety and risk for substance dependence among late adolescents/young adults. J Anxiety Disord 19(3):275-294. https://doi.org/10.1016/j.janxd is.2004.03.001

6. Pratt LA, Druss BG, Manderscheid RW, Walker ER (2016) Excess mortality due to depression and anxiety in the United States: results from a nationally representative survey. Gen Hosp Psychiatry 39(1):39-45. https://doi.org/10.1016/j.genhosppsych. 2015.12.003

7. Trautmann S, Rehm J, Wittchen HU (2016) The economic costs of mental disorders. EMBO Rep 17(9):1245-1249. https://doi.org/ 10.15252/embr.201642951

8. Collishaw S (2015) Annual research review: secular trends in child and adolescent mental health. J Child Psychol Psychiatry 56(3):370-393. https://doi.org/10.1111/jcpp.12372 
9. Merikangas KR (2018) Time trends in the global prevalence of mental disorders in children and adolescents: gap in data on U.S. youth. J Am Acad Child Adolesc Psychiatry 57(5):306-307. https://doi.org/10.1016/j.jaac.2018.03.002

10. Bitsko RH, Holbrook JR, Ghandour RM et al (2018) Epidemiology and impact of health care provider-diagnosed anxiety and depression among US children. J Dev Behav Pediatr 39(5):395403. https://doi.org/10.1097/DBP.0000000000000571

11. Duffy ME, Twenge JM, Joiner TE (2019) Trends in mood and anxiety symptoms and suicide-related outcomes among US undergraduates, 2007-2018: evidence from two national surveys. J Adolesc Health 65(5):590-598. https://doi.org/10.1016/j.jadoh ealth.2019.04.033

12. Goodwin RD, Weinberger AH, Kim JH, Wu M, Galea S (2020) Trends in anxiety among adults in the United States, 2008-2018: rapid increases among young adults. J Psychiatr Res 130:441-446. https://doi.org/10.1016/j.jpsychires.2020.08.014

13. Calling S, Midlöv P, Johansson S-E, Sundquist K, Sundquist J (2017) Longitudinal trends in self-reported anxiety. Effects of age and birth cohort during 25 years. BMC Psychiatry 17(1):119. https://doi.org/10.1186/s12888-017-1277-3

14. Sigfusdottir ID, Asgeirsdottir BB, Sigurdsson JF, Gudjonsson GH (2008) Trends in depressive symptoms, anxiety symptoms and visits to healthcare specialists: a national study among Icelandic adolescents. Scand J Public Health 36(4):361-368. https://doi.org/ $10.1177 / 1403494807088457$

15. Keyes KM, Nicholson R, Kinley J et al (2014) Age, period, and cohort effects in psychological distress in the United States and Canada. Am J Epidemiol 179(10):1216-1227. https://doi.org/10. 1093/aje/kwu029

16. Twenge JM, Cooper AB, Joiner TE, Duffy ME, Binau SG (2019) Age, period, and cohort trends in mood disorder indicators and suicide-related outcomes in a nationally representative dataset, 2005-2017. J Abnorm Psychol 128(3):185-199. https://doi.org/ 10.1037/abn0000410

17. Bor W, Dean AJ, Najman J, Hayatbakhsh R (2014) Are child and adolescent mental health problems increasing in the 21 st century? A systematic review. Aust N Z J Psychiatry 48(7):606-616. https://doi.org/10.1177/0004867414533834

18. Ross A, Kelly Y, Sacker A (2017) Time trends in mental wellbeing: the polarisation of young people's psychological distress. Soc Psychiatry Psychiatr Epidemiol 52(9):1147-1158. https://doi. org/10.1007/s00127-017-1419-4

19. Grills AE, Ollendick TH (2002) Issues in parent-child agreement: the case of structured diagnostic interviews. Clin Child Fam Psychol 5(1):57-83. https://doi.org/10.1023/a:1014573708569

20. Kessler RC, Berglund P, Demler O, Jin R, Merikangas KR, Walters EE (2005) Lifetime prevalence and age-of-onset distributions of DSM-IV disorders in the National Comorbidity Survey Replication. Arch Gen Psychiatry 62(6):593-602. https://doi.org/10. 1001/archpsyc.62.6.593

21. Zahn-Waxler C, Shirtcliff EA, Marceau K (2008) Disorders of childhood and adolescence: gender and psychopathology. Annu Rev Clin Psychol 4(1):275-303. https://doi.org/10.1146/annurev. clinpsy.3.022806.091358

22. Becerra-Culqui TA, Liu Y, Nash R et al (2018) Mental health of transgender and gender nonconforming youth compared with their peers. Pediatrics 141(5):e20173845. https://doi.org/10.1542/peds. 2017-3845/-/DCSupplemental

23. Fergusson DM, Horwood LJ, Beautrais AL (1999) Is sexual orientation related to mental health problems and suicidality in young people? Arch Gen Psychiatry 56(10):876-880. https://doi.org/10. 1001/archpsyc.56.10.876

24. Reisner SL, Vetters R, Leclerc M et al (2015) Mental health of transgender youth in care at an adolescent urban community health center: a matched retrospective cohort study. J Adolesc
Health 56(3):274-279. https://doi.org/10.1016/j.jadohealth.2014. 10.264

25. McLaughlin KA, Hilt LM, Nolen-Hoeksema S (2007) Racial/ ethnic differences in internalizing and externalizing symptoms in adolescents. J Abnorm Child Psychol 35(5):801-816. https://doi. org/10.1007/s10802-007-9128-1

26. Children, Youth and Families (2011) Youth services—department of human services-Government of Dane County, Wisconsin. https://danecountyhumanservices.org/yth/dcyc.aspx. Published 5 Jan 2011. Accessed 28 Nov 2019

27. U.S. Census Bureau, American Community Survey 2018 American Community Survey 1-Year Estimates, Table DP05; generated by Katharine Parodi; using data.census.gov; <https://data. census.gov/cedsci/> Accessed 9 May 2021

28. Wisconsin Department of Public Instruction. WISEdash Public Portal. https://wisedash.dpi.wi.gov/Dashboard/dashboard/16840. Accessed 9 May 2021

29. Kroenke K, Spitzer RL, Williams JBW, Monahan PO, Löwe B (2007) Anxiety disorders in primary care: prevalence, impairment, comorbidity, and detection. Ann Intern Med 146(5):317-325. https://doi.org/10.7326/0003-4819-146-5-200703060-00004

30. Plummer F, Manea L, Trepel D, McMillan D (2016) Screening for anxiety disorders with the GAD-7 and GAD-2: a systematic review and diagnostic metaanalysis. Gen Hosp Psychiatry 39:2431. https://doi.org/10.1016/j.genhosppsych.2015.11.005

31. Staples LG, Dear BF, Gandy M et al (2019) Psychometric properties and clinical utility of brief measures of depression, anxiety, and general distress: the PHQ-2, GAD-2, and K-6. Gen Hosp Psychiatry 56:13-18. https://doi.org/10.1016/j.genhosppsych.2018. 11.003

32. Cioffredi L-A, Kamon J, Turner W (2021) Effects of depression, anxiety and screen use on adolescent substance use. Prev Med Rep 22:101362. https://doi.org/10.1016/j.pmedr.2021.101362

33. Ghandour RM, Sherman LJ, Vladutiu CJ, Ali MM, Lynch SE, Bitsko RH, Blumberg SJ (2019) Prevalence and treatment of depression, anxiety, and conduct problems in US children. J Pediatr 206:256-267.e3. https://doi.org/10.1016/j.jpeds.2018.09.021

34. Ruscio AM, Hallion LS, Lim CCW et al (2017) Cross-sectional comparison of the epidemiology of DSM-5 generalized anxiety disorder across the globe. JAMA Psychiat 74(5):465. https://doi. org/10.1001/jamapsychiatry.2017.0056

35. R Development Core Team (2019) R: A language and environment for statistical computing. Vienna, Austria. https://www.Rproject.org/.

36. Weinberger AH, Gbedemah M, Martinez AM, Nash D, Galea S, Goodwin RD (2018) Trends in depression prevalence in the USA from 2005 to 2015: widening disparities in vulnerable groups. Psychol Med 48:1308-1315. https://doi.org/10.1017/S003329171 7002781

37. Robinson JP, Espelage DL (2011) Inequities in educational and psychological outcomes between LGBTQ and straight students in middle and high School. Educ Res 40(7):315-330. https://doi.org/ 10.3102/0013189X11422112

38. Robinson-Cimpian JP (2014) Inaccurate estimation of disparities due to mischievous responders: several suggestions to assess conclusions. Educ Res 43(4):171-185. https://doi.org/10.3102/00131 89X14534297

39. Keyes KM, Gary D, O'Malley PM, Hamilton A, Schulenberg J (2019) Recent increases in depressive symptoms among US adolescents: trends from 1991 to 2018. Soc Psychiatry Psychiatr Epidemiol 54(8):987-996. https://doi.org/10.1007/ s00127-019-01697-8

40. Mojtabai R, Olfson M, Han B (2016) National trends in the prevalence and treatment of depression in adolescents and young adults. Pediatrics 138(6):e20161878. https://doi.org/10.1542/peds. 2016-1878 
41. Plemmons G, Hall M, Doupnik S et al (2018) Hospitalization for suicide ideation or attempt: 2008-2015. Pediatrics 141(6):e20172426. https://doi.org/10.1542/peds.2017-2426

42. Olfson M, Blanco C, Wang S, Laje G, Correll CU (2014) National trends in the mental health care of children, adolescents, and adults by office-based physicians. JAMA Psychiat 71(1):81-90. https://doi.org/10.1001/jamapsychiatry.2013.3074

43. American Psychological Association (2018) Stress in America: Generation Z. Stress in America Survey.

44. Benner AD, Wang Y, Shen Y, Boyle AE, Polk R, Cheng Y-P (2018) Racial/ethnic discrimination and well-being during adolescence: a meta-analytic review. Am Psychol 73(7):855-883. https:// doi.org/10.1037/amp0000204

45. Clark TC, Lucassen MFG, Bullen P et al (2014) The health and well-being of transgender high school students: results from the New Zealand Adolescent Health Survey (Youth'12). J Adolesc Health 55(1):93-99. https://doi.org/10.1016/j.jadohealth.2013.11. 008

46. Eisenberg ME, Gower AL, McMorris BJ, Rider GN, Shea G, Coleman E (2017) Risk and protective factors in the lives of transgender/gender nonconforming adolescents. J Adolesc Health 61(4):521-526. https://doi.org/10.1016/j.jadohealth.2017.04.014

47. Mustanski BS, Garofalo R, Emerson EM (2010) Mental health disorders, psychological distress, and suicidality in a diverse sample of lesbian, gay, bisexual, and transgender youths. Am J Public
Health 100(12):2426-2432. https://doi.org/10.2105/AJPH.2009. 178319

48. Alegría M, Green JG, McLaughlin KA, Loder S (2015) Disparities in child and adolescent mental health and mental health services in the US New York: William T. Grant Foundation

49. Cheng TL, Goodman E (2015) Committee on pediatric research. Race, ethnicity, and socioeconomic status in research on child health. Pediatrics 135(1):225. https://doi.org/10.1542/peds. 2014-3109

50. Gassman-Pines A, Ananat EO, Fitz-Henley J (2020) COVID-19 crisis impacts on parent and child psychological well-being. Pediatrics. https://doi.org/10.1542/peds.2020-007294

51. Huckins JF, DaSilva AW, Wang W et al (2020) Mental health and behavior of college students during the early phases of the COVID-19 pandemic: longitudinal smartphone and ecological momentary assessment study. J Med Internet Res 22(6):e20185. https://doi.org/10.2196/20185

52. Alegría M, NeMoyer A, Bagué IF, Wang Y, Alvarez K (2018) Social determinants of mental health: where we are and where we need to go. Curr Psychiat Rep 20(11):95. https://doi.org/10.1007/ s11920-018-0969-9

53. Velasco-Mondragon E, Jimenez A, Palladino-Davis AG, Davis D, Escamilla-Cejudo JA (2016) Hispanic health in the USA: a scoping review of the literature. Public Health Rev. https://doi. org/10.1186/s40985-016-0043-2 